\title{
CARBON DISCLOSURE PROJECT (CDP): CARACTERIZAÇÃO DA EVIDENCIAÇÃO DE INFORMAÇÕES AMBIENTAIS DAS EMPRESAS BRASILEIRAS ENTRE 2006 E 2010
}

\author{
Luana das Graças Queiróz Farias ${ }^{\mathrm{a}}$ \\ José Célio Silveira Andrade \\ Maria de Fátima Barbosa Góes ${ }^{a}$ \\ Ricardo Luiz Naves Rabêlo Filho ${ }^{\text {a }}$ \\ ${ }^{a}$ Universidade Federal da Bahia (UFBA), Salvador, BA, Brasil
}

\section{Resumo}

Nos últimos anos, o tema mudanças climáticas tem recebido especial atenção das empresas brasileiras e de seus stakeholders e, nesse contexto, o Carbon Disclosure Project (CDP) vem desempenhando um papel importante na evidenciação de informações ambientais. Esta pesquisa buscou analisar as principais características do disclosure de informações ambientais das empresas brasileiras respondentes à solicitação do CDP entre 2006 e 2010. Dessa forma, as características foram analisadas a partir da delimitação de seis categorias: status, nível de responsabilidade, riscos, estratégias gerenciais, oportunidades e contabilização de GEE. Para consecução do objetivo proposto, adotou-se a pesquisa descritiva e, para levantamento dos dados empíricos secundários, extraídos dos questionários respondidos pelas empresas brasileiras publicados no website do CDP, utilizou-se a pesquisa documental. As técnicas de análise de conteúdo e estatística descritiva foram utilizadas para tratamento dos dados. Com relação às características do disclosure ambiental apresentadas, dentre os resultados econtrados, enfatizam-se os seguintes indicadores: (1) status: revelou que, apesar de ainda não ser uma prática da maioria das empresas participantes do CDP, o nível de disclosure vem aumentando significativamente; (2) nível de responsabilidade: demonstrou que o tema está sendo tratado prioritariamente em âmbito de conselho adminstrativo ou órgão executivo, ou seja, na mais alta instância corporativa; (3) riscos: conforme esperado, foi o indicador que apresentou maior número de sentenças quantitativas, tendo cerca de 1/3 das empresas destacado os riscos físicos e regulatórios; (4) oportunidades: quase 36\% das empresas informaram que consideram a participação no mercado de crédito de carbono como a oportunidade mais importante gerada pelas mudanças climáticas; (5) estratégias gerenciais: 30,5\% das empresas evidenciaram a adoção de iniciativas estratégicas relacionadas às mudanças climáticas, como utilização de metodologias de quantificação de GEE, programa de sequestro de carbono e quantificação de CO2, enquanto 20,42\% informaram a implantação de SGAs e de programas ambientais e de responsabilidade social; (6) contabilização de GEE: confirmando as expectativas, mais de 1/3 das empresas informaram que o GHG Protocol foi a metodologia selecionada para fazer seu inventário de emissões de GEE. De forma geral, alguns setores têm empreendido mais ações para aprimorar seus conhecimentos sobre riscos, oportunidades e estratégias gerenciais. Nessa pesquisa, sobretudo, notou-se que os segmentos utilidade pública, consumo e financeiro apresentaram forte representação nos aspectos referentes ao número de empresas respondentes à solicitação do CDP e à quantidade de informações ambientais fornecidas sobre os indicadores pesquisados. Finalmente, este estudo apontou, também, que, apesar das empresas brasileiras participantes do CDP apresentarem um disclosure parcial, elas já começaram a identificar a necessidade de aumentar a transparência e a comunicação com seus stakeholders. Ao mesmo tempo, as mais engajadas na questão ambiental começam a reconhecer o papel do CDP no enfrentamento.

Palavras-chave: CDP, Mudanças Climáticas, Brasil 


\section{INTRODUÇÃO}

A mudança climática global constitui-se no mais sério desafio para as políticas ambientais das próximas décadas. Cada vez mais estudos científicos demonstram que o aquecimento global representa uma ameaça real ao mundo e às organizações, exigindo novos modelos, políticas e atenção dos governos, das empresas, da sociedade e uma maior eficácia da Governança Ambiental Global (GAG) para o enfrentamento desse problema (MINISTÉRIO DA CIÊNCIA E TECNOLOGIA, 2008).

Em particular, para as empresas, a constituição de ações e de iniciativas para atenuar esse problema engloba um contexto de riscos e de oportunidades, perpassando por questões de melhoria de desempenho, incorporação de novos padrões e, sobretudo, aperfeiçoamento das suas práticas de disclosure ou evidenciação ambiental, visando conciliar os interesses econômicos com a preservação ambiental (HOFFMAN, 2005; HOFFMAN \&WOODY, 2008; KIM, 2008).

Para Cormier et al. (2004), o disclosure ambiental é considerado um conjunto de informação comunicado aos grupos de interesse ou stakeholders através da publicação de relatórios ambientais e de sustentabilidade anuais para que as organizações adquiram legitimidade. Nessa mesma perspectiva, Borges et al. (2010) observam que a evidenciação de práticas sociais e ambientais responsáveis pode constituir-se num fator de agregação de valor à organização, visto que, além de atuar na promoção da transparência das informações para todos os seus stakeholders, ajuda no cumprimento da legislação.

Em âmbito mundial, sobretudo nas duas últimas décadas, a apresentação da evidenciação ambiental e social das grandes companhias tem crescido em complexidade e tamanho (GRAY et al., 2001). No Brasil, a evidenciação ambiental é um problema real das organizações e ainda pouco explorado no campo acadêmico, porém tem havido evolução da quantidade e qualidade das informações "verdes" disponíveis nos relatórios anuais. Esses relatórios e as demonstrações contábeis são ferramentas importantes utilizadas pelas empresas para evidenciação dos seus objetivos e dos meios realizados para alcançá-los (MURCIA et al., 2008; RIBEIRO, 2005).

Assim, as pressões para maior disclosure ambiental, originadas por diferentes atores - a exemplo das ONGs, fornecedores, governos, empregados, consumidores e comunidade - tem aumentado ano a ano. Porém, recentemente, um novo grupo de stakeholders vem exercendo forte influência nesse assunto: os bancos, os investidores e as seguradoras (HOFFMAN, 2005).

Isto posto, dentre as diversas iniciativas internacionais para evidenciação das informações ambientais, tem-se o Carbon Disclosure Project (CDP). Trata-se de uma entidade internacional sem fins lucrativos que detém o maior banco de dados sobre as ações e respostas das empresas para enfrentamento do problema das mudanças climáticas, visando à promoção de diálogos entre investidores e corporações através da abertura de informação (CDP, 2010; PATTBERG \& ENECHI, 2009 apud ANDRADE, 2009).

A divulgação dessa iniciativa no Brasil iniciou em 2005, mas a primeira edição nacional do CDP ocorreu em 2006. No país, o CDP vem construindo um banco de dados corporativo sobre as estratégias das empresas para reduzirem suas emissões de Gases de Efeito Estufa (GEE), tendo como maiores apoiadores investidores signatários nacionais, principalmente fundos de pensão (CDP, 2010). Até 2009, as empresas brasileiras selecionadas para participarem do CDP eram as pertencentes ao índice Brasil 80. A partir de 2010, as empresas selecionadas foram escolhidas através do índice da IBrX da BM\&F Bovespa.

Diante dessa perspectiva, surge a questão que norteou a presente pesquisa: "Quais são as principais características do disclosure ambiental das empresas brasileiras participantes do Carbon Disclosure Project no período de 2006 a 2010?" Visando responder à pergunta de pesquisa, o objetivo geral deste artigo pode ser definido como se segue: analisar as principais características do disclosure de informações ambientais das empresas brasileiras respondentes à solicitação do CDP entre 2006 e 2010. 
Para atingir este propósito, os procedimentos metodológicos adotados foram as pesquisas bibliográfica e documental. Os dados empíricos secundários foram obtidos através de pesquisa documental nos questionários respondidos pelas empresas brasileiras publicados no website do CDP. As técnicas de análise de conteúdo e de estatística descritiva foram utilizadas para tratamento dos dados.

Por fim, a estrutura do artigo é composta por três partes, além desta introdução e das considerações finais: na primeira, é apresentado o constructo teórico; na segunda, são detalhados os procedimentos metodológicos utilizados e, na terceira, é feita a apresentação e discussão dos resultados.

\section{CONSTRUCTO TEÓRICO}

Esta seção apresenta o constructo teórico que sustenta o desenvolvimento deste artigo e está subdividida em dois itens: (i) Principais respostas das empresas para o enfrentamento das mudanças climáticas e (ii) Disclosure ou Evidenciação ambiental.

\subsection{Principais respostas das empresas para o enfrentamento das mudanças climáticas}

Em âmbito mundial, as mudanças climáticas vêm emergindo como uma das principais preocupações dos governos, empresas, investidores e sociedade em geral. A intensificação de concentração de GEE na atmosfera é resultante, de forma primária, do aumento das atividades humanas (CNI, 2011).

As mudanças climáticas afetarão diretamente a economia, levando a transformações prioritariamente estruturais (CDP, 2010). Nesse contexto, passam a incidir riscos climáticos sobre as dinâmicas competitivas das empresas. Os riscos climáticos - que podem se tornar o fator mais significativo para a dinâmica competitiva de muitas corporações - são classificados por Hoffman e Woody (2008) e Kolk et al. (2008) como sistêmicos, que compreendem as categorias de: riscos físicos, regulatórios e reputacionais. O website do CDP corrobora a classificação dos autores supracitados quanto às denominações dos riscos, mas acrescenta também a categoria de riscos mercadológicos representados pela redução na demanda por produtos intensivos em energia e no aumento dos custos no uso dos processos intensivos em energia.

Setores como o agrícola, securitário, imobiliário e turístico são confrontados com riscos de natureza física, influenciados diretamente pelo aumento das temperaturas e do nível do mar; já os setores intensivos em combustíveis fósseis estão sujeitos a riscos legais e reputacionais (KOLK et al., 2008).

De forma geral, os estudos demonstram que as respostas das empresas às alterações climáticas são bastante diversificadas, predominando abordagens político-institucionais e focadas na estratégia de mercado. Na primeira, as corporações devem agir a partir de um contexto político-institucional, empreendendo esforços para influenciar a configuração e a direção do processo de negociação internacional (KOLK \& PINKSE, 2004; HILLMAN et al., 1999; EBERLEIN \& MATTEN, 2009; ANDRADE \& COSTA, 2008). Já para a segunda abordagem, as respostas são econômicas e dizem respeito ao macroambiente, microambiente e ambiente interno dos negócios, sendo as alterações climáticas consideradas a questão mais proeminente da sustentabilidade (REID \& TOFFEL, 2009; FUCHS, 2008; KIM, 2008; HOFFMAN \& WOODY, 2008; SUSSMAN \& FREED, 2008). Fuchs (2008), por exemplo, afirma que as empresas realmente engajadas com as mudanças climáticas estão deslocando o foco de uma postura baseada no gerenciamento de riscos para uma que prioriza as oportunidades de negócios criadas e, consequentemente, gera vantagens competitivas num ambiente de negócios com restrições ao uso do carbono.

Hoffman (2005) sistematizou sete oportunidades para as empresas que desejam enfrentar as mudanças climáticas, conforme descrito no quadro abaixo: 
Quadro 01- Oportunidades para as empresas enfrentarem as mudanças climáticas

\begin{tabular}{|l|l|}
\hline \multicolumn{1}{|c|}{ OPORTUNIDADE } & \multicolumn{1}{|c|}{ DETALHAMENTO } \\
\hline Melhoria operacional & $\begin{array}{l}\text { Corresponde à melhoria dos processos e produtos, resultando } \\
\text { na redução de custos de energia, utilização de energia limpa } \\
\text { e renovável, dentre outros. }\end{array}$ \\
\hline $\begin{array}{l}\text { Antecipação e influência sobre } \\
\text { as regulamentações de mudanças } \\
\text { climáticas }\end{array}$ & $\begin{array}{l}\text { Englobam questões sobre a legislação, acordos e exigências } \\
\text { que afetam a taxa de emissão de GEE pelas organizações. }\end{array}$ \\
\hline Acesso a novas fontes de capital & $\begin{array}{l}\text { Abrange iniciativas voltadas para a redução das taxas e para } \\
\text { fomento ao uso de tecnologias limpas. }\end{array}$ \\
\hline Elevação da reputação corporativa & $\begin{array}{l}\text { Inclui os ganhos de reputação e imagem decorrentes das } \\
\text { ações públicas associadas às estratégias climáticas. }\end{array}$ \\
\hline Novasoportunidadesmercadológicas & $\begin{array}{l}\text { Compreendem levantamentos de novas oportunidades no } \\
\text { mercado. }\end{array}$ \\
\hline $\begin{array}{l}\text { Melhoria da gestão de recursos } \\
\text { humanos }\end{array}$ & $\begin{array}{l}\text { Envolve planos de carreira, retenção de talentos, salários, } \\
\text { benefícios, confiança e desempenho superior de seus } \\
\text { funcionários. }\end{array}$ \\
\hline
\end{tabular}

Para enfrentar os desafios e explorar as oportunidades advindas das mudanças climáticas, as empresas devem constantemente diagnosticar questões econômicas, políticas, sociais, científicas e técnicas, visando consolidar uma estratégia pró-ativa (JABBOUR \& SANTOS, 2009). Essas oportunidades, por sua vez, estão sendo geradas a partir da condução de políticas corporativas de controle de emissão de GEE nas áreas de geração de energia renovável, participações no mercado de carbono, incentivos econômicos fiscais, desenvolvimento de novos produtos e serviços de baixa emissão, tecnologias limpas e novos nichos de mercado (CDP, 2010).

Por outro lado, Hoffman \& Woody (2008) defendem que os impactos das mudanças climáticas ainda estão se manifestando e, para tanto, faz-se necessário que as organizações desenvolvam estratégias climáticas, orientadas por três passos: a) conhecimento sobre as suas emissões de carbono e o impacto de transição de mercado sobre seus produtos e serviços, incluindo as escolhas de ferramentas para medir a emissão, sistemas de gerenciamento de dados, registro das emissões e avaliação; b) decisão acerca das ações para reduzir emissões e avaliar oportunidades de negócio, englobando ações de competência organizacional, como qualificação da liderança, desenvolvimento de equipes interfuncionais e equipes de eficiência energética, estabelecimento de metas e objetivos, dentre outras e c) participação no processo de desenvolvimento de políticas voltadas para enfrentar as mudanças climáticas, numa tentativa de influenciar as regras do jogo.

Acrescenta-se, ainda, nesse contexto, a necessidade de institucionalização da divulgação de informações padronizadas como um componente central da GAG (CDP, 2010; KOLK et al., 2008). Trata-se de um canal de comunicação da empresa com seus diversos stakeholders, capaz de influenciar no seu desempenho, nas ações de mitigação dos impactos ambientais negativos e nos padrões organizacionais de comparação através do benchmarking.

Os investidores estão valorizando os aspectos inerentes ao modelo de gestão ambiental e responsabilidade social empresarial adotado para combater as mudanças climáticas. Deste modo, algumas iniciativas voluntárias têm sido introduzidas para fomentar, instituir e mensurar a emissão de GEE (KOLK et al., 2008; PATTBERG \& ENECHI, 2009 apud ANDRADE, 2009). O próprio CDP - Carbon Disclosure Project, organização sem fins lucrativos cujo objetivo é divulgar informações sobre as emissões de GEE e a gestão de carbono implementada pelas empresas, é um exemplo destas iniciativas. 
Na verdade, o CDP é um dos principais regimes não públicos de GAG desenvolvidos pelo setor privado para responder ao problema das mudanças climáticas (PATTBERG \& ENECHI, 2009 apud ANDRADE, 2009). Com a finalidade de analisar as tendências dos riscos e oportunidades decorrentes das mudanças climáticas, as informações requeridas pelo CDP concentram-se em quatro áreas principais: a) visão da Administração sobre riscos e oportunidades que a mudança climática representa para os negócios; b) contabilização das emissões de gases de efeito estufa; c) estratégia gerencial de redução das emissões ou minimização de riscos e capitalização, e d) governança corporativa relativa às mudanças climáticas.

O CDP abrange um grupo de 534 investidores institucionais globais - responsáveis pela gestão de US\$ 64 trilhões - que buscam investir em empresas socialmente responsáveis. No que tange à participação das empresas brasileiras no projeto, vale destacar que, desde a primeira publicação brasileira, em 2006, houve ampliação no número de questionários preenchidos ao longo dos anos, conforme exposto no Quadro 02.

Quadro 02 - Evolução da participação das empresas brasileiras no CDP.

\begin{tabular}{|c|c|c|}
\hline CDP - BRASIL & PARTICIPANTES TOTAIS & NÃO RESPONDENTES \\
\hline 2006 & 50 & 7 \\
\hline 2007 & 60 & 3 \\
\hline 2008 & 75 & 1 \\
\hline 2009 & 80 & 0 \\
\hline 2010 & 80 & 3 \\
\hline
\end{tabular}

No Brasil, os fundos de pensão, seguidos pelos bancos e seguradoras, são os maiores investidores institucionais do CDP. De acordo com a ordem cronológica, o BrasilPrev Seguros e Previdências S. A. foi o primeiro ente brasileiro signatário do pedido de disclosure, seguido pela Associação Brasileira das Entidades Fechadas de Providência Complementar (ABRAPP), Associação Nacional dos Bancos de Investimento (ANBID), Banco do Brasil e Previ (CDP, 2010).

Os resultados apresentados pelo CDP (2010) destacaram que a participação de instituições brasileiras vem aumentando ao longo dos anos, com a adesão de 55 signatários nacionais (representando 11\% dos 534 signatários globais) e sinalizando que a conscientização corporativa sobre os riscos climáticos está crescendo. Além disso, as corporações brasileiras apresentam altos índices de respostas, classificando-se em 2010 como quarto melhor índice de respostas do mundo (CDP, 2010).

\subsection{Disclosure ou Evidenciação Ambiental}

As questões relativas às preocupações sociais e ambientais das empresas têm provocado o aumento das pesquisas na área de contabilidade social e ambiental. De maneira especial, a pesquisa sobre abertura de informações, evidenciação ambiental ou disclosure tem apresentado grande importância. A evidenciação das informações ambientais pode ser apresentada de diversas formas como, por exemplo, demonstrações contábeis, relatórios de gestão, guias e respostas de questionários demandados pelos seus stakeholders, entre outras.

Os estudos atuais nesta área são marcados por três características, a saber: a) natureza empírica, abordando o comportamento da evidenciação de informações de caráter ambiental; b) abordagem do assunto em conjunto com o disclosure social; c) evidenciação ambiental das informações empresariais como resultado da pressão originada pelos stakeholders, especialmente se a organização desenvolve algum tipo de atividade potencialmente poluidora. 
Diversos autores estão colaborando para identificar os significados e os objetivos do disclosure ambiental no contexto empresarial. Dentre eles, Borges et al. (2010) afirmam que a expressão "evidenciação ambiental" está sendo utilizada no sentido de aperfeiçoar a comunicação da empresa; Tinoco e Kraemer (2004) argumentam que seu propósito é disseminar informações dos desempenhos econômico, financeiro, social e ambiental das instituições aos stakeholders. Já para Salotti e Yamamoto (2005) e Borges et al. (2010), respectivamente, a importância do tema está associada à percepção dos investidores quanto ao risco que a companhia apresenta e à divulgação de todas as informações que possam afetar o patrimônio da empresa.

Para Paiva (2003, p.5), as empresas estão buscando estreitar seu relacionamento com os usuários das informações ambientais e, por isso, têm "procurado atender aos requisitos ambientais, sendo a evidenciação a forma de torná-los públicos".

Complementando essas questões, Rosa e Ensslin (2010) asseguram que a prestação de informações aos investidores quanto às práticas ambientais e a maior evidenciação dessas informações pelas empresas pode vir a atrair mais investidores. Ou seja, trata-se de informações que revelam os modelos de gestão de negócios e ambiental da organização, a exemplo dos aspectos: político-institucionais; dados patrimoniais; resultados financeiros; uso e consumo de recursos naturais; contabilização de emissões de GEE; impactos ambientais; responsabilidade socioambiental, entre outros.

O Quadro 03 abaixo reúne as principais pesquisas nacionais sobre o tema, segundo a apreciação de Bellen et al. (2007).

Quadro 03 - Principais pesquisas nacionais sobre disclosure ambiental

\begin{tabular}{|l|l|}
\hline \multicolumn{1}{|c|}{ AUTORES } & \multicolumn{1}{c|}{ ABORDAGEM SOBRE DISCLOSURE } \\
\hline $\begin{array}{l}\text { Rover et al. (2009) } \\
\text { Lima (2007) }\end{array}$ & $\begin{array}{l}\text { Voltada para o tratamento dos dados pelas empresas, incluindo } \\
\text { principalmente as informações sobre externalidades e passivos } \\
\text { ambientais. }\end{array}$ \\
\hline Nossa (2002) & $\begin{array}{l}\text { Dedicada aos estudos comparativos de disclosure entre as empresas do } \\
\text { setor de papel e celulose de diversos países. }\end{array}$ \\
\hline Ribeiro (2005) & $\begin{array}{l}\text { Estudo comparativo do conteúdo de disclosure ambiental no setor de } \\
\text { papel e celulose no Brasil e nos Estados Unidos. }\end{array}$ \\
\hline $\begin{array}{l}\text { Lindstaed \& Ott } \\
(2007)\end{array}$ & $\begin{array}{l}\text { Confrontamento dos regulamentos ambientais entre o Brasil e os Estados } \\
\text { Unidos. }\end{array}$ \\
\hline Costa e Marion (2007) & $\begin{array}{l}\text { Avaliação da uniformidade das informações ambientais evidenciadas em } \\
\text { relatórios de várias empresas brasileiras. }\end{array}$ \\
\hline Calixto et al. (2007) & $\begin{array}{l}\text { Investigação das diferenças entre os canais de divulgação e o tipo de } \\
\text { informações evidenciadas por esses. }\end{array}$ \\
\hline
\end{tabular}

Segundo Nossa (2002), a participação no disclosure ambiental pode ser realizada de forma obrigatória ou voluntária. No Brasil, as normas contábeis referentes ao disclosure ambiental são de caráter não obrigatório. A divulgação ambiental, entretanto, é orientada pelos seguintes preceitos: a) parecer normativo $\mathrm{n}^{\circ} 15187$ da Comissão de Mobiliária de Valores (CMV); b) norma e procedimento de auditoria $\mathrm{n}^{\mathrm{o}} 11$, estabelecido pelo Instituto dos Auditores Independentes do Brasil e c) Resolução n ${ }^{0}$ 1003/04, do Conselho Federal de Contabilidade, que confirmou a norma Brasileira de Contabilidade Técnica ${ }^{\circ} 15$. Essas resoluções confirmam o papel da contabilidade ambiental na avaliação dos eventos e transações econômico-financeiras relacionadas ao meio ambiente, ao mesmo tempo, permitindo o levantamento de informações que auxiliem o gestor na tomada de decisões e, consequentemente, contribuam para o lucro e o desenvolvimento sustentável (RIBEIRO, 2005; FERREIRA, 2003). 


\section{ASPECTOS METODOLÓGICOS}

O presente estudo foi orientado nos aspectos metodológicos pelos objetivos e procedimentos técnicos empregados. Esta pesquisa, em relação aos objetivos, pôde ser classificada como de natureza descritiva, pois se baseou nas características do disclosure ambiental apresentadas pelas empresas que disponibilizaram suas respostas no website do CDP (www.cdproject.net) no período de 2006 a $2010^{1}$.

Para a coleta dos dados, foram utilizados os procedimentos de pesquisa bibliográfica e documental. No que concerne ao levantamento literário, foi realizada uma pesquisa bibliográfica enfocando os seguintes conteúdos: respostas das empresas para o enfrentamento das mudanças climáticas, Carbon Disclosure Project $(C D P)$ e disclosure ou evidenciação ambiental. Quanto à pesquisa documental, foi usada a análise de conteúdo, proposta por Bardin (2000) para exame das respostas das empresas brasileiras publicadas no website do CDP.

Dessa forma, os procedimentos metodológicos do estudo foram constituídos por três fases operacionais interdependentes, a saber: a) primeira etapa: análise de conteúdo individualizada das respostas publicadas pelas empresas unicamente no website do CDP, objetivando levantar as principais informações sobre o disclosure ambiental apresentado pelas empresas e a classificação das mesmas por setor produtivo; b) segunda etapa: delimitação das categorias analíticas da pesquisa e c) terceira etapa: análise das categorias identificadas nas respostas das empresas e tratamento dos dados utilizando-se de estatística descritiva através de análise de frequência.

As categorias analíticas selecionadas nesta pesquisa foram baseadas nos questionários padrões enviados pelo CDP às empresas e na literatura. Sob essa perspectiva, as categorias escolhidas foram: status, nível de responsabilidade, riscos, oportunidades, estratégias gerenciais e contabilização de GEE.

O universo da pesquisa representa as empresas brasileiras que receberam a solicitação do disclosure pelo CDP no período de 2006 a 2010 e que efetivamente disponibilizaram seus questionários respondidos no website, totalizando 81. Para fins deste estudo, estas empresas foram agrupadas em onze setores produtivos, conforme descritos a seguir:

Quadro 04 - Distribuição das empresas investigadas por setor produtivo.

\begin{tabular}{|l|c|}
\hline \multicolumn{1}{|c|}{ SETOR PRODUTIVO } & \% PARTICIPAÇÃO \\
\hline Consumo não-cíclico e cíclico & 23,46 \\
\hline Utilidade pública & 17,28 \\
\hline Financeiro & 14,81 \\
\hline Construção e transporte & 11,11 \\
\hline Telecomunicações & 8,64 \\
\hline Materiais Básicos Madeira/ Papel & 7,41 \\
\hline Materiais básicos/siderurgia/ metalurgia & 6,17 \\
\hline Indústria de bens/equipamentos & 3,70 \\
\hline Petróleo \& gás/ biocombustível e combustíveis & 3,70 \\
\hline Materiais básicos /mineração /minerais metálicos & 2,47 \\
\hline Materiais básicos químico/ petroquímico & 1,23 \\
\hline
\end{tabular}

1 O ano de 2005 não foi incluso na pesquisa porque não houve publicação da edição nacional, havendo apenas atividades de divulgação do projeto no país. 


\section{APRESENTAÇÃO E DISCUSSÃO DOS RESULTADOS}

Nesta seção, são apresentadas as análises realizadas quanto aos indicadores: status, nível de responsabilidade, riscos, oportunidades, estratégias gerenciais e contabilização de GEE, utilizando como base analítica a estatística descritiva e as medições das respostas em frequência (número de ocorrências).

Em todos os indicadores, com exceção do "status", foi realizada análise de frequência. Deste modo, a análise de conteúdo desta pesquisa foi feita por meio de leitura e interpretação das respostas publicadas pelas empresas no website do CDP e seguindo-se de forma quantitativa, a partir da categorização e quantificação (contagem da frequência). O total, respectivamente, de categorias e frequência por cada indicador correspondeu a: 12; 118 (para o nível de responsabilidade); 6; 231 (para os riscos); 11; 114 (para oportunidades); 8; 84 (para estratégias gerenciais) e 6; 141 (para contabilização de GEE).

Além disso, os resultados demonstraram que houve uma forte participação de três setores produtivos (consumo, utilidade pública e financeiro), destacando-se principalmente nos aspectos de número de empresas participantes, quantidade de respostas publicadas (nível de disclosure), nível de responsabilidade e contabilização de GEE, expostos a seguir:

\section{- Status}

Verificou-se, através do indicador status, o percentual de empresas que efetivamente publicaram suas respostas no website no período de 2006 a 2010. Neste período, manteve-se constante a quantidade de empresas analisadas, totalizando oitenta e uma. Sendo assim, o número de empresas que evidenciaram efetivamente seu nível de disclosure ambiental aumentou, o que pode ser percebido através do status "VIR" na Figura 01 abaixo, cujos percentuais ao logo dos anos de 2006 a 2010 foi: $0 \%, 38 \%, 35 \%, 35 \%$ e $41 \%$.

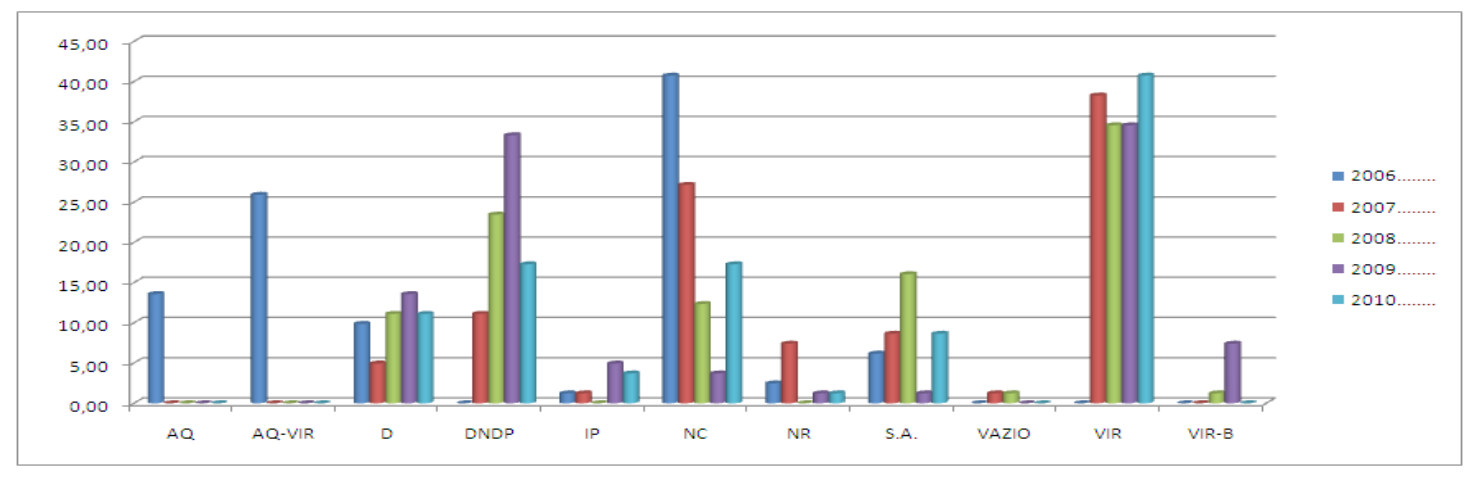

Figura 01- Evidenciação das informações ambientais sobre status das empresas respondentes ao CDP.

Legenda: AQ: questionário respondido; AQ/VIR: questionário respondido/verificar resposta do investidor; D: declinou; DNDP: dados não disponíveis para publicação; IP/VIR: informações providenciadas/ verificar resposta do investidor; NC: não consta resposta publicada no website; SA: verificar outra empresa; Não informou dado acerca deste indicador; VIR: verificar resposta do investidor.

Assim, o ano de 2010 revelou os maiores números de disclosure e de empresas participantes em relação aos demais anos investigados, corroborando os resultados obtidos no relatório do CDP, edição 2010. Contudo, de forma geral, os resultados demonstraram que a evidenciação ambiental não foi uma prática da maioria das empresas participantes do CDP. Assim, pôde-se inferir que o nível de disclosure foi parcial, aproveitando pouco os benefícios proporcionados enquanto mecanismo de comunicação e divulgação do desempenho ambiental das empresas aos seus grupos de interesse. 
- $\quad$ Nível de Responsabilidade

O nível de responsabilidade corresponde à instância institucional à qual o tema da mudança climática foi alocado pelas empresas respondentes à solicitação do CDP. Para análise desse item, utilizaram-se onze categorias, a saber: comissão designada; comitê; departamento; diretoria; gerência; grupo de trabalho; grupo interno; instituto e gerência; órgão executivo/conselho administrativo; todos os funcionários e não informou resposta (a frase anterior está sem sentido). Dessa forma, a única categoria que se destacou foi o órgão executivo/conselho administrativo (44,07\%), conforme mostrado na Figura 02 abaixo.

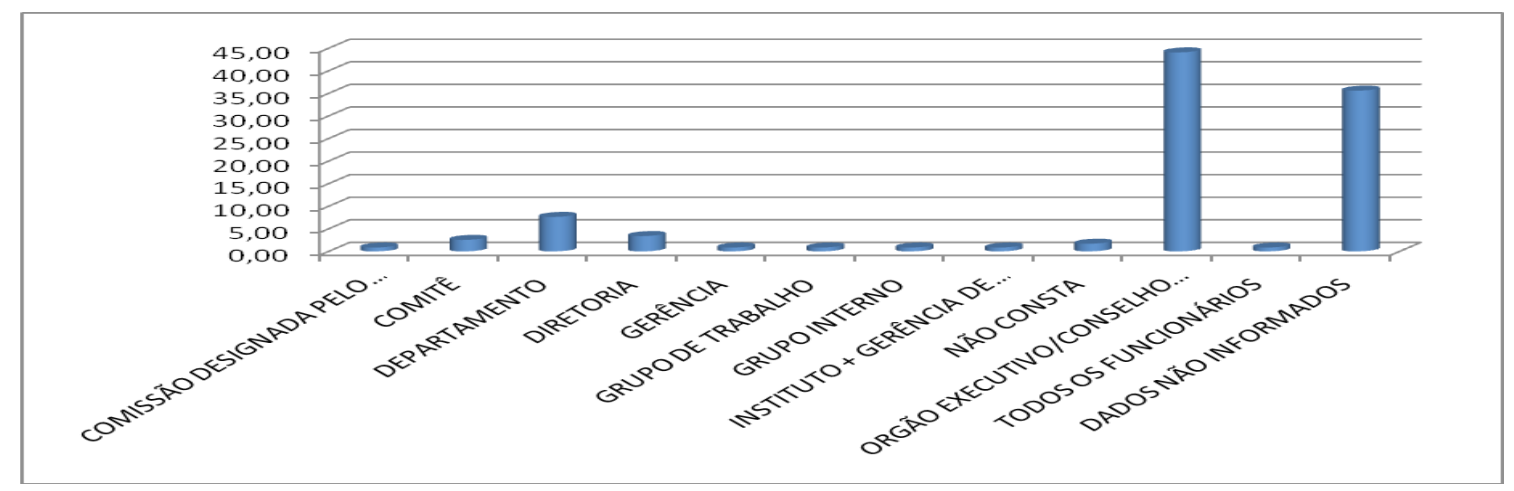

Figura 02 - Evidenciação das informações sobre nível de responsabilidade das empresas respondentes ao CDP.

Esse resultado reforça que o tema está sendo tratado nos altos escalões corporativos, um dos fatores mais importantes para o sucesso de qualquer estratégia climática (HOFFMAN \& WOODY, 2008; CDP, 2010). Portanto, as questões relacionadas às mudanças climáticas estão sendo introduzidas na agenda corporativa das empresas brasileiras participantes do CDP, ao mesmo tempo em que o interesse dos acionistas pela questão está crescendo, inserindo gradualmente os impactos da mudança climática nas decisões sobre competitividade e retorno dos investimentos.

\section{- $\quad$ Riscos}

Os riscos decorrentes das mudanças climáticas estão sendo analisados sob uma perspectiva de negócio e investimento, visto que os stakeholders já começam a pressionar as corporações para que estas divulguem suas exposições a esses riscos. Determinadas empresas e setores sofrem impactos maiores do que outros, pois as implicações das mudanças climáticas não são uniformes e tem impactos diferentes sobre os setores produtivos.

Nesse estudo, os riscos mais colocados em destaque pelas empresas em ordem crescente de citações foram os físicos $(35,06 \%)$, regulatórios $(29,00 \%)$ e mercadológicos $(18,45 \%)$, havendo predomínio dos físicos e dos regulatórios, conforme Figura 03.

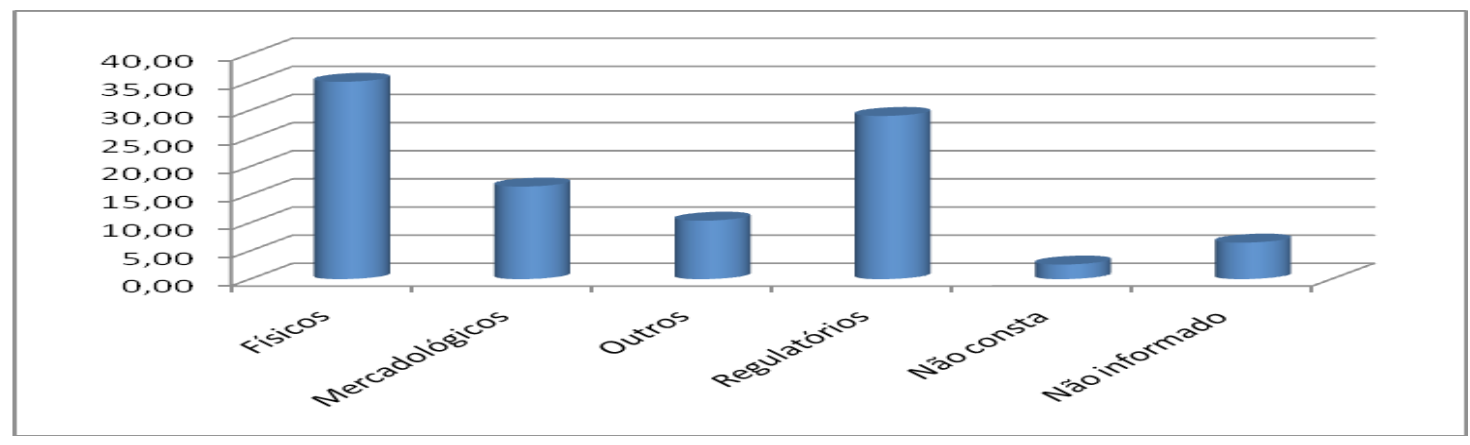

Figura 03 - Evidenciação das informações ambientais sobre riscos das empresas respondentes ao CDP. 
Isso pode estar ocorrendo, todavia, pelo fato das catástrofes naturais, das alterações de temperatura e do aumento da concentração de GEE na atmosfera serem identificados pelas empresas como fatores que podem afetar os ativos físicos, a cadeia de suprimentos, ou a própria estrutura do negócio. E, por sua vez, os regulatórios, segundo Hoffman e Woody (2008) e a CNI (2011), têm muita notoriedade no ambiente empresarial, uma vez que definem as emissões de GEE e as regras do jogo, delimitando o contorno da vantagem competitiva empresarial e favorecendo determinadas ações, empresas e setores para manter certo controle sobre o ambiente de negócios futuros.

A respeito dos riscos impostos pelas mudanças climáticas alterarem conforme o setor de atuação das organizações, os achados dessa pesquisa indicam que os setores de utilidade pública, consumo e financeiro apresentaram maior ocorrência em relação aos riscos identificados.

\section{- $\quad$ Oportunidades}

Muitas empresas estão percebendo as mudanças climáticas como uma oportunidade, em detrimento da antiga visão de ameaça. De forma geral, estas empresas estão buscando oportunidades ligadas ao mercado de créditos de carbono, novas habilidades e tecnologias que reduzam as suas emissões.

Os resultados relativos ao indicador oportunidades demonstram que a maioria das empresas respondentes ao CDP vislumbrou a participação no mercado de crédito de carbono (35,9\%), sobretudo no Mecanismo de Desenvolvimento Limpo (MDL) como a principal oportunidade gerada pelas mudanças climáticas para o ambiente corporativo. Isso se justifica, em boa parte, pela compreensão das organizações em relação aos benefícios gerados pelo mercado de carbono e a atuação das empresas além do contexto de suas operações, a exemplo do próprio MDL.

Esse resultado coaduna-se com a posição do Brasil no ranking dos países em desenvolvimento que tem mais projetos de MDL no mundo, com 7\% dos projetos de MDL, perdendo somente para a China e Índia com, respectivamente, $38 \%$ e 27\% (MINISTÉRIO DA CIÊNCIA E TECNOLOGIA, 2011).

Dentre as categorias que apresentaram maior frequência depois do MDL, estão o desenvolvimento de produtos, serviços e processos com preocupação ambiental, com frequência de $21,05 \%$, e a antecipação e influência sobre as ações regulatórias nas mudanças climáticas, com 10,53\%. Neste último caso, para obter maior poder de influência sobre as novas regras para o período pós-Kyoto, as empresas estão atuando mais ativamente na GAG do clima através de participação em acordos e parcerias público/privado.

As alterações climáticas revelam oportunidades para os diferentes setores produtivos. Contudo, levando em consideração as três oportunidades mais citadas, o setor de utilidade pública contribuiu sozinho com 15,79\% das sentenças ambientais referentes ao MDL. No caso dos demais setores, a maior participação foi representada pelos segmentos construção e transporte, materiais básicos químico/petroquímico, materiais básicos/mineração/minerais metálicos, perfazendo cada um o total de 10,53\% das citações apresentadas sobre oportunidades.

\section{- $\quad$ Estratégias Gerenciais}

O gerenciamento de riscos, a identificação de novas oportunidades de negócios e o aumento do conhecimento sobre o tema faz com que as empresas priorizem suas estratégias gerenciais, enfatizando a criação de vantagem competitiva. A literatura analisada sobre a matéria, entretanto, afirma que não existe uma estratégia dominante, mas um grande portfólio de medidas que podem ser implementadas para reduzir as emissões de GEE da organização e atender aos requisitos impostos pela economia de baixo carbono. Essas alternativas envolvem medidas no processo de gestão (mudanças nas operações, otimização do uso de energia, substituição de viagens por videoconferências) até mudanças no processo produtivo (substituição de fontes de 
energia não renováveis por fontes renováveis e utilização de matérias primas menos intensivas em carbono).

Conforme pode ser visto na Figura 04, as estratégias gerenciais identificadas neste estudo estão focadas nas seguintes ações: iniciativas ambientais relacionadas às mudanças climáticas (inventário, redução de GEE, metodologias de GEE, programa de sequestro de carbono, quantificação de $\mathrm{CO}_{2}$ ), 30,5\%; outras informações ambientais (políticas ambientais, SGA, programas ambientais, responsabilidade social), 20,42\%; e desenvolvimento de produtos, serviços e processos com preocupação ambiental (gestão de processos, novos produtos e serviços sustentáveis, redução de custos operacionais etc..), 16,67\%.

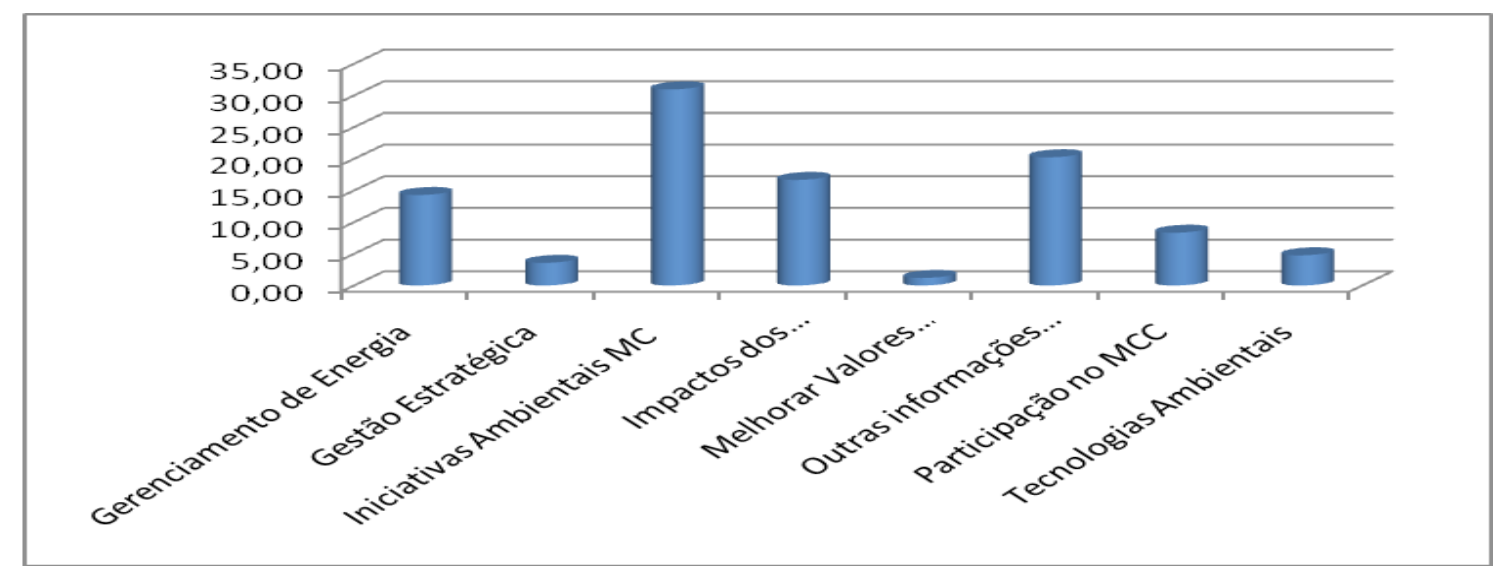

Figura 04 - Evidenciação das informações sobre estratégias gerenciais das empresas respondentes ao CDP.

Quanto à participação dos setores produtivos, notou-se que as principais estratégias gerenciais identificadas envolveram um número expressivo de setores, totalizando oito. Ainda abordando a participação setorial neste indicador, verificou-se que o setor de utilidade pública apresentou $19 \%$ de todas as citações ambientais evidenciadas neste indicador.

\section{- Contabilização de GEE}

Algumas corporações e investidores estão se movendo em direção à nova economia de baixo carbono e, consequentemente, estão mais preocupados com a medição e a avaliação das suas emissões de GEE. A necessidade de quantificar as emissões corporativas de GEE está se configurando como uma tendência mundial. Nesse contexto, as empresas estão coletando informações com intuito de entender o perfil das suas emissões, ou seja, as fontes e a intensidade dessas emissões (HOFFMAN \& WOODY, 2008; CNI, 2011).

As emissões de GEE podem ser contabilizadas por meio de metodologias para calcular a quantidade de dióxido de carbono emitido para a atmosfera. Reconhece-se, mundialmente, que a ferramenta mais utilizada pelas empresas para o seu gerenciamento de GEE é o modelo Greenhouse Gas Protocol (GHG). Essa metodologia foi desenvolvida pelo World Business Council for Sustainable Development (WBCSD) em conjunto com World Resources Institute (WRI) e seu objetivo é classificar as emissões em três tipos: escopo 1: emissões diretas (em geral, incluem emissões originadas pelos processos produtivos na empresa); escopo 2: emissões indiretas (equivalem às energias compradas, emissões geradas pelo uso dos produtos da empresa, pelo transporte do material, viagens a negócios e deslocamento de pessoal); e escopo 3: outras emissões indiretas de fontes (envolvem outras emissões que são consequência das atividades diretas da empresa, mas ocorrem em fontes não controladas pela empresa, como a queima de combustível proveniente das viagens aéreas dos funcionários) (CDP, 2011).

Conforme esperado neste estudo, o padrão mais citado pelas empresas para contabilização de GEE foi o modelo GHG (38,30\%). Porém, outras metodologias e padrões de referência foram também citados pelas empresas para medir ou estimar suas emissões. Dentre eles, estão os baseados nas instituições: ISO e IPCC (Figura 04). 
Para efeito de comparação entre os setores, identificou-se que os setores de utilidade pública, consumo e financeiro, respectivamente, registram maior número de citações. Todavia, verificou-se uma maior participação do setor de utilidade pública. Assim, o fato das empresas que compõem o setor de utilidade pública fazerem uso de insumos como água e energia intensivamente faz com que elas naturalmente estejam mais expostas à pressão para redução de GEE.

\section{CONSIDERAÇÕES FINAIS}

O aumento da concentração de GEE na atmosfera está provocando mudanças climáticas nos ecossistemas naturais e nos ambientes competitivos das empresas. Nesse contexto, o CDP vem tratando de aspectos específicos dos impactos das mudanças climáticas relacionados à sustentabilidade nos negócios, mobilizando empresas em âmbito mundial e organizando bancos de dados sobre o disclosure de informações ambientais.

Este artigo teve como objetivo analisar as principais características do disclosure de informações ambientais das empresas brasileiras respondentes à solicitação do CDP entre 2006 e 2010. A amostra do estudo foi representada pelas empresas brasileiras que efetivamente disponibilizaram seus questionários respondidos neste período, revelando que, nos quatro anos analisados, o ano de 2010 evidenciou o maior número de empresas participantes e, por conseguinte, maior nível de disclosure ambiental.

Com relação às características do disclosure ambiental apresentadas, dentre os resultados econtrados, enfatizam-se os seguintes indicadores: (1) status: revelou que, apesar de ainda não ser uma prática da maioria das empresas participantes do CDP, o nível de disclosure vem aumentando significativamente - de nenhuma empresa em 2006 a 41\% das empresas em 2010. Assim, pôde-se inferir que o nível de disclosure foi parcial; (2) nível de responsabilidade: demonstrou que o tema está sendo tratado prioritariamente em âmbito de conselho adminstrativo ou órgão executivo, ou seja, na mais alta instância corporativa, considerado um dos fatores decisivos para o sucesso de qualquer estratégia climática; (3) riscos: conforme esperado, foi o indicador que apresentou maior número de sentenças quantitativas, tendo cerca de 1/3 das empresas destacado os riscos físicos e regulatórios; (4) oportunidades: quase 36\% das empresas informaram que consideram a participação no mercado de crédito de carbono, sobretudo no Mecanismo de Desenvolvimento Limpo (MDL), como a oportunidade mais importante gerada pelas mudanças climáticas para o ambiente empresarial; (5) estratégias gerenciais: $30,5 \%$ das empresas evidenciaram a adoção de iniciativas estratégicas relacionadas às mudanças climáticas, como uilização de metodologias de quantificação de GEE, programa de sequestro de carbono e quantificação de $\mathrm{CO}_{2}$, enquanto 20,42\% informaram a implantação de SGAs e de programas ambientais e de responsabilidade social; (6) contabilização de GEE: confirmando as expectativas, mais de 1/3 das empresas informaram que o GHG Protocol foi a metodologia selecionada para fazer seu inventário de emissões de GEE.

De forma geral, alguns setores têm empreendido mais ações para aprimorar seus conhecimentos sobre riscos, oportunidades e estratégias gerenciais. Nessa pesquisa, sobretudo, notou-se que os segmentos utilidade pública, consumo e financeiro apresentaram forte representação nos aspectos referentes ao número de empresas respondentes à solicitação do CDP e à quantidade de informações ambientais fornecidas sobre os indicadores pesquisados.

Por fim, este estudo apontou, também, que, apesar das empresas brasileiras participantes do CDP apresentarem um disclosure parcial, elas já começaram a identificar a necessidade de aumentar a transparência e a comunicação com seus stakeholders. Ao mesmo tempo, as mais engajadas na questão ambiental começam a reconhecer o papel do CDP no enfrentamento das mudanças do clima e a perceber as oportunidades de uma nova economia de baixo carbono. 


\section{AGRADECIMENTOS}

Os autores gostariam de agradecer ao Conselho Nacional de Desenvolvimento Científico e Tecnológico $(\mathrm{CNPq})$ pelo suporte financeiro para esta pesquisa.

\section{REFERÊNCIAS}

ANDRADE, J. C. S. O papel das empresas brasileiras participantes do Carbon Disclosure Project na governança ambiental global do clima. Bolsa de Produtividade em Pesquisa CNPq. UFBA, 2009.

ANDRADE, J. C. S.; COSTA, P. O. Mudança Climática, Protocolo de Kyoto e Mercado de Créditos de Carbono: desafios à governança ambiental global. O\&S, v.15, n.45, 2008.

BARDIN, L. Análise de conteúdo. Lisboa: Edições 70, 2000

BELLEN, H. M. V.; GALLON, A. V; SOUZA, F. C; ROVER, S. Produção Científica e Perspectivas Teóricas da Área Ambiental: Um Levantamento a Partir dos Artigos Publicados em Congressos e Periódicos Nacionais da Área de Contabilidade e Administração. In: Congresso USP de Contabilidade e Controladoria, 7., 2007, São Paulo. Anais... São Paulo: USP, 2007. CD-ROM.

BORGES, A. P; ROSA, F. S; ENSSLIN, S. R. Evidenciação das práticas ambientais: um estudo nas grandes empresas brasileiras de papel e celulose. Produção, v. 20, n. 3, jul./set. 2010.

CALIXTO, Laura; BARBOSA, Ricardo Rodrigues; LIMA, Marilene Barbosa. Disseminação

de informações ambientais voluntárias: relatórios contábeis versus internet. Revista Contabilidade \& Finanças (on line), vol. 18, 2007.

CARBON DISCLOSURE PROJECT (CDP). Relatório 2010. Brasil. Disponível em: http://< www. cdproject.net $/$ CDPResults/CDP_2009-Brazil_Report>. Acesso em: 10 de dezembro. 2010.

. Relatório 2009. Brasil. Disponível em: http://<www.cdproject.net $/$ CDPResults/ CDP_2009-Brazil_Report>. Acesso em: 10 de dezembro. 2010.

. Relatório 2008. Brasil. Disponível em: http://<www.cdproject.net $\backslash /$ CDPResults/ CDP_2009-Brazil_Report>. Acesso em: 10 de dezembro. 2010.

. Relatório 2007 .Brasil. Disponível em: http://<www.cdproject.net $/$ CDPResults/ CDP_2009-Brazil_Report>. Acesso em: 10 de dezembro. 2010.

. Relatório 2006. Brasil. Disponível em: http://<www.cdproject.net $\backslash / C D P R e s u l t s /$ CDP_2009-Brazil_Report>. Acesso em: 10 de dezembro. 2010.

.(CDP). Respostas das empresas respondentes à solicitação do CDP 2006. Disponível em: http://<www.cdproject.net /CDPResults/CDP_2009-Brazil_Report>. Acesso em: 10 de dezembro. 2010.

(CDP). Respostas das empresas respondentes à solicitação do CDP 2007. Disponível em :http://<www.cdproject.net /CDPResults/CDP_2009-Brazil_Report>. Acesso em: 10 de dezembro. 2010.

.(CDP) Respostas das empresas respondentes à solicitação do CDP 2008. Disponível em:. http://<www.cdproject.net $\backslash /$ CDPResults/CDP_2009-Brazil_Report>. Acesso em: 10 de dezembro. 2010. 
.(CDP). Respostas das empresas respondentes à solicitação do CDP 2010. Disponível em: http://<www.cdproject.net>. Acesso em: 25 de jan. 2011.

CONFEDERAÇÃO NACIONAL DA INDÚSTRIA (CNI). Estratégias Corporativas de Baixo Carbono: Gestão de Riscos e Oportunidades. Confederação Nacional da Indústria. Brasília, 2011.

CORMIER, D. et al. Corporate environmental disclosure: contrasting management's perceptions with reality. Journal of Business Ethics, v. 49, n. 2, 2004.

COSTA, R; MARION, J. A uniformidade na evidenciação das informações

ambientais. Revista de Contabilidade e Finanças. v.43, 2007.

EBERLEIN, B.; MATTEN, D. Business Responses to Climate Change Regulation in Canada and Germany: Lessons for MNCs from Emerging Economies. Journal of Business Ethics, v. 86, n. 2, march, 2009.

FERREIRA, A. C. S. Contabilidade ambiental: uma informação para o desenvolvimento sustentável. São Paulo: Atlas, 2003.

FUCHS, P. R. Estratégias Climáticas das Empresas Brasileiras: Investigação nos setores de Papel e Celulose e Automotivo com Base em Benchmarks Internacionais. Dissertação (Programa de Pós- Graduação em Administração). PUC-Rio, Rio de Janeiro, 2008.

GRAY, R. et al. Social and environmental disclosure and corporate characteristics: a research note and extension. Journal of Business Finance \& Accounting, v. 28, n. 12001.

HILLMAN, L. B.; ZARDKOOHI, J.; ASGHAR. Corporate Political Strategies and Firm Performance: Indications Of Firm-Specific Benefits From Personal Service In The U.S. Government. Strategic Management Journal, v. 20,1999.

HOFFMAN, A. J. Climate Change Strategy: The Business Logic behind Voluntary Greenhouse Gas Reductions, California Management Review. v. 47, n. 3, 2005.

HOFFMAN, A. J.; WOODY, J. G. Climate change: what's your business strategy? (Memo to the CEO). Harvard Business School Press, Cambridge, MA, 15 April, 2008.

JABBOUR, J. C. C.; SANTOS, F. A. C. Sob os ventos da mudança climática: desafios, oportunidade se o papel da função produção no contexto do aquecimento global, 2009. Disponível em: $h t t p: / /<w w w$. scielo.br/scielo.php?pid=S0104-530X2009000100011\&sc.>Acesso em: 10 de agosto. 2010

KIM, Y. Corporate Responses to Climate Change: The Resource-Based View, 2008. Disponível em: http://<www.oikos-international.org/fileadmin/oikos-international/in>Acesso em 01 março. 2010.

KOLK, A; LEVY, D; PINKSE, J. Corporate Responses in an Emerging Climate Regime: The Institutionalization and Commensuration of Carbon Disclosure. European Accounting Review, v. 17, n. 4, 2008. n. 3, 2004 .

KOLK, A.; PINKSE, J. Market strategies for climate change. European Management Journal, v. 22,

LIMA, D. V.; VIEGAS, W.. Tratamento contábil e evidenciação das externalidades ecológicas. Revista de contabilidade e finanças da USP, n. ${ }^{\circ}$ 30, 2002.

LINDSTAEDT , Â. R. S., OTT, E. Evidenciação de Informações Ambientais pela Contabilidade: um estudo comparativo entre as normas internacionais

(ISAR/UNCTAD), norte americanas e brasileiras. Contabilidade Vista \& Revista, v. 18, n. 4,,out./ dez. 2007. 
MINISTÉRIO DA CIÊNCIA E TECNOLOGIA. Status atual das atividade s de projeto no âmbito do Mecanismo de Desenvolvimento Limpo no Brasil e no Mundo (2008). In: Mudanças Climáticas. Disponível

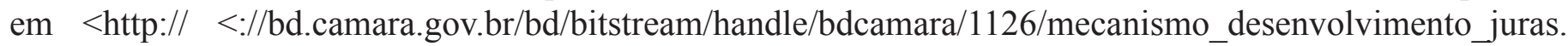
pdf? sequence=1>. Acesso em: 28 jan. 2011.

MURCIA, F.; SANTOS, A.; SALOTTI, B.; NASCIMENTO, A. Mapeamento da pesquisa sobre disclosure ambiental no cenário internacional: uma revisão dos artigos publicados em periódicos de língua inglesa no período de 1997-2007. In: Congresso USP de Controladoria e Contabilidade, 8., 2008, São Paulo Anais ... São Paulo: FEA/USP, 2008. CD- ROM.

NOSSA, V. Disclosure ambiental: uma análise do conteúdo dos relatórios ambientais de empresas do setor de papel e celulose em nível internacional. 2002. 246. f. Tese (Doutorado em Ciências Contábeis) - Universidade de São Paulo, São Paulo, 2002.

PAIVA, P. R. Contabilidade Ambiental: evidenciação dos gastos ambientais com transparência focada na preservação. São Paulo: Atlas, 2003.

REID, E. M; TOFFEL, M, W. Responding to Public and Private Politics: Corporate Disclosure of Climate Change Strategies. Forthcoming in Strategic Management Journal, June 16, 2009.

RIBEIRO, M. S. Contabilidade Ambiental. São Paulo: Saraiva, 2005.

ROSA, F. S.; ENSSLIN, L. Evidenciação Ambiental: Processo Estruturado de Revisão de Literatura sobre Avaliação de Desempenho da Evidenciação Ambiental. Sociedade, Contabilidade e Gestão (UFRJ), v. 4, 2010.

ROVER, S; BORBA, J. A; DELRI MURCIA, F. Características do Disclosure Ambiental de Empresas Brasileiras Potencialmente Poluidoras: Análise das demonstrações Financeiras e dos Relatórios de Sustentabilidade do período de 2005 a 2009. Contextus: Revista Contemporânea de Economia e Gestão. v. 7, nº 1, jan-jun, 2009.

SALOTTI, B. M.; YAMAMOTO, M. M. Ensaio sobre a teoria da divulgação. Brazilian Business Review, v. 2, n. 1, 2005.

SUSSMAN, F.; G; FREED, J. R. Adapting to climate change: a business approach. Prepared for the Pew Center on global climate change,2008. Disponível em: http $\backslash:<$. www.pewclimate.org/docUploads/ Business-Adaptation.pdf >Acesso em: 05 de abril. 2010.

TINOCO, J. E. P; KRAEMER, M. E. P. Contabilidade e Gestão Ambiental. São Paulo: Atlas, 2004. 


\title{
CARBON DISCLOSURE PROJECT (CDP): CHARACTERIZATION OF THE DISCLOSURE OF ENVIRONMENTAL INFORMATION OF BRAZILIAN COMPANIES FROM 2006 TO 2010
}

\begin{abstract}
In recent years, the theme climate changing has received special attention from Brazilian companies and their stakeholders. In this context, the Carbon Disclosure Project (CDP) has played an important role in the disclosure of environmental information. This research sought to examine the main features of the disclosure of environmental information and Brazilian companies responding to the request of CDP between 2006 and 2010. Thus, by this way, they were analyzed from the definition of six categories: status, level of responsibility, risks, management strategies, opportunities and accounting of GHG. In order to achieve our goal, we adopted the descriptive research method, and for empirical surveys of secondary data, filled out by the Brazilian companies and published in the website of the CDP, we used the documental research method for the analysis of questionnaires. The techniques of content analysis and descriptive statistics were used for data processing. Regarding the characteristics of environmental disclosure that were shown, among the results were found, we emphasized the following indicators: (1) status: revealed that, although it is not a common practice among the companies that answered the CDP questionnaire, the level of disclosure has been increased considerably; (2) level of responsibility: demonstrated that the issue is being given priority in the context of the board administrator/executive body. It means within the higher corporate level; (3) risk: as was expected, this indicator showed the largest number of quantitative sentences, with around 1/3 of the companies emphasizing the physical and regulatory risks; (4) opportunities: almost 36\% of companies reported that the participation in the carbon credit market was considered the most important opportunity for businesses created by climate changes; (5) management strategies: $30.5 \%$ of the companies highlighted initiatives in relation to climate changing like the use of methodologies of quantification of GEE, kidnapping carbon programs and CO2 quantification, while 20.42\% reported the implementation of SGAs and environmental and social responsibility programs; (6) accounting of GEE: confirming the expectations, $38.30 \%$ of companies reported that the GHG Protocol methodology was selected to make its inventory of greenhouse gas emissions. In general, some industries have undertaken more actions to improve their knowledge about risks, opportunities and management strategies. In this research, especially, it was noted that the segments Public Utility, Consumer and Financial had strong representation on issues relating to the number of companies responding to CDP's request and the amount of environmental information provided on the indicators measured. Finally, this study found that, despite the Brazilian companies participating in the CDP make a partial disclosure, they have begun to identify the need for increased transparency and communication among its stakeholders and at the same time, they recognize the importance of the CDP to withstand the effects of climate changes and have begun to recognize the opportunities are rising from a world economy based on low carbon.
\end{abstract}

KeyWords: CDP; Climate Change, Brazil 